(EULAR) Social Leagues + International Organisation of Youth with Rheumatism (IOYR).

\section{SP0047 ASSESSING THE NEEDS OF THE INDIVIDUAL}

N Price. Locomotor Directorate, North Staffordshire Hospitals NHS Trust, Stoke on Trent, UK

10.1136/annrheumdis-2001.7

The shift away from service driven to needs lead service delivery will be explored.

The needs of the individual during the phases of disease progress will compare perceived versus actual. Contribution of the multidisciplinary team at all stages will be highlighted.

The move to patient/individual self-efficacy and the effect on relationships with health professionals provide an arena for potential conflict. The health professionals perception of the individuals needs are often not compatible with the actual needs of the individual.

The results of an in depth interview, with a patient, will explore their needs and whether they have been met. (An interview audited tool will be used).

Financial constraints, shortage of health professionals, the political agenda, compliance of the individual and "patient power" will highlight the areas of unmet needs.

The needs of carers and service providers are often neglected at the expense of the "individual". The conflict of the carer and individual will also be addressed.

Does the individual know best?

\section{REFERENCES \\ 1 Arthritis Impact Measurement Scales (AIMS) \\ 2 Health Assessment Questionnaire (HAQ) \\ 3 SF - 36 Health Survey \\ 4 The NHS and Community Care Act 1990}

\section{SP0049 RAISING PRIORITY FOR THE NEEDS OF THE INDIVIDUAL}

AD Woolf. Department of Rheumatology, Royal Cornwall Hospital, Truro, UK

\subsection{6/annrheumdis-2001.8}

Musculoskeletal conditions (MSC) have an enormous and growing impact recognised by WHO Europe in "Health 21" yet national health care priorities in most European countries do not include them. The aim of the Bone and Joint Decade is to redress this imbalance by advocacy supported by evidence. To improve the outcome for the individual greater priority has to be given to the provision of existing proven interventions that meet the needs and wishes of the person with a MSC, and better interventions need to be developed through research. Education of public, people with MSC, health care providers, politicians and health professionals is fundamental to changing the negative perception of what can be achieved and in improving standards of care. Any campaign to meet these objectives needs to be supported by relevant data of the impact of MSC and evidence based solutions to reduce this. Key messages that highlight the issues, priorities and these solutions need to be developed for the decision-makers and any other relevant audiences. These messages should be conveyed by the powerful alliance of those with MSC and those who provide their care. The potent image of advocates must be used to attract media attention that is central to the strength of any campaign. Finally targets must be set to measure the success of any campaign. The Bone and Joint Decade is identifying the burden of MSC with the WHO and developing evidence based strategies for prevention with the support of the EU. National and local campaigns are necessary that use this evidence to achieve change and fulfil the aim of the Decade, which is to improve the health-related quality of life for those with musculoskeletal conditions.

\section{T-Cell activation and MHC - Thursday 14 June, 16.00-17.30/South Hall}

\section{SP0050 THE ROLE OF HLA IN RHEUMATOID ARTHRITIS: INDUCTION OF AGGRESSION OR FAILURE TO PROTECT?}

${ }^{1} \mathrm{R}$ De Vries, ${ }^{1} \mathrm{E}$ Zanelli, ${ }^{2} \mathrm{~F}$ Breedveld. ${ }^{1}$ Immunohematology and Blood Transfusion, Leiden, The Netherlands; ${ }^{2}$ Rheumatology, Leiden University Medical Center, Leiden, The Netherlands

\subsection{6/annrheumdis-2001.9}

Genetic factors contribute both to the development and the course of Rheumatoid Arthritis (RA). The contribution of inherited genetic variability has been estimated $60 \%$. Moreover, stochastic processes during the development of the $\mathrm{T}$ and $\mathrm{B}$ lymphocytes repertoire or so-called somatic genetic diversity may be responsible for an as yet unknown fraction of the remaining $40 \%$ of the factors contributing to the pathogenesis of RA.

Thus far, the only known inherited genetic factors are encoded by the HLA system. These factors contribute about $40 \%$ of the inherited genetic variability and also contribute to the somatic genetic diversity. The main contribution of the HLA system is conferred by the HLA class II region and HLA-DR genes are certainly involved but HLA-DQ genes also may have a contribution. Perhaps most importantly: certain HLA-DR genes may provide dominant protection. HLA genes not only contribute to the development but also the course and severity of RA. There is also evidence for dominant protection against the development of erosions. We observed that this effect may be relatively short-lasting and overruled by a very early start of treatment with DMARDS (Yard et al., submitted for publication). These observations suggest that pharmacogenomics may offer possibilities for a tailor-made optimal treatment for individual RA patients. The standard hypothesis for an association of an HLA class II allele with an auto-immune disease is that autoreactive $\mathrm{T}$ cells recognise certain self-epitopes that are exclusively or preferentially presented by that particular HLA class II allele. The Shared Epitope (SE) hypothesis (Gregersen et al. 1987) is a paradigm of this. More than 20 years of intensive research along these lines have however yielded little evidence for this concept. Therefore, we have developed an alternative hypothesis. In this hypothesis auto-reactive $\mathrm{T}$ cells do not play a role in the initiation but in the regulation of the disease process (Zanelli et al., Rheumatology, in press). Our model is an extension of the one proposed by Thomas and Lipsky (Immunology Today 1996; 17: 559-560). It starts with activation of synoviocytes by a trauma or an infection. Usually this results in a local inflammation that resolves in a few days, but in the presence of one or more (yet to be identified) genetic factors (nót HLA class II) an aberrant activation of synoviocytes results in a chronic inflammation. As proposed by Thomas and Lipsky chemo-attraction of dendritic 